\title{
EFFECT OF POSTOPERATIVE EPIDURAL ANALGESIA AFTER MAJOR ORTHOPEDIC SURGERIES, A RETROSPECTIVE STUDY
}

\author{
Laxmi Pathak ${ }^{1}$
}

\section{ABSTRACT}

INTRODUCTION: Epidural analgesia has been used in many major surgeries like upper abdominal surgery, cardiothoracic surgery, orthopedic surgery etc. Epidural analgesia is one of the commonly used methods of postoperative pain control despite its associated complications. So, this study was conducted to find out its effectiveness in major orthopedic surgeries done in Universal College of Medical Sciences.

MATERIALAND METHODS: A retrospective study was done at Universal College of Medical Sciences \& Teaching Hospital, Bhairahawa, Nepal from July 2012 to June 2014. Data of 57 patients, aged between 17 to 91 years having American Society of Anesthesiologists (ASA) physical status 1 and 2 who had undergone major orthopedic surgeries under spinal anesthesia and lumber epidural catheterization were collected and important information regarding anesthesia and surgery, epidural catheter and postoperative epidural analgesia, any complications if occurred throughout the study period were recorded. Departmental Protocol for epidural analgesia was followed in these patients. Patients who received intraoperative epidural anesthesia or analgesia and any other anesthetic or analgesic agents were excluded in this study.

RESULTS: This study found epidural analgesia, a very effective way to relieve pain in patients undergoing major orthopedic surgeries, when given according to the Departmental Protocol. There were no any complications related to epidural analgesia till $4^{\text {th }}$ postoperative day. Out of 57, only 2 patients received injection Ketorolac intravenously as a rescue analgesic at the same day of operation before epidural top up. Average time to demand for analgesic after the last dose of epidural top up was 21.933 hours. All patients were mobilized around their beds on $2^{\text {nd }}$ postoperative day. The average postoperative days of hospital stay was only 6.5 days.

CONCLUSION: Epidural mixture of Bupivacaine-morphine in lower dose and concentration given as an intermittent bolus dosing via lumber epidural catheter is safe and very effective in relieving postoperative pain after major orthopedic surgeries without any significant complications.

KEYWORDS: Bupivacaine-morphine mixture; Lumber Epidural Analgesia; Postoperative pain relief

1. Associate Professor, Department of Anesthesiology, Universal College of Medical Sciences \& Teaching Hospital, Bhairahawa, Nepal

\author{
For Correspondence \\ Dr. Laxmi Pathak \\ Associate Professor, \\ Department of Anesthesiology, \\ Universal College of Medical Sciences \& Teaching \\ Hospital, Bhairahawa, Nepal \\ E-mail: laxmi_pathak22@yahoo.com
}




\section{INTRODUCTION}

Pain not only stimulates sympathetic activity but also affects patient's psychology. Inadequately controlled postoperative pain may immobilize the patients that ultimately prolongs the duration of hospital stay and increases the cost.

Role of epidural analgesia is well known. It is very effective in relieving intraoperative or postoperative pain after major upper abdominal, thoracic or orthopedic surgeries. The side effects or complications might be related to procedure or the drug used like dural perforation, epidural hematoma, infection, urinary retention, hypotension, pruritus and respiratory depression. Thus, this study was conducted to find out the effectiveness of postoperative epidural analgesia by collecting the data of patients who underwent major orthopedic procedures.

\section{MATERIAL AND METHODS}

Data of total 57 patients underwent elective major orthopedic surgeries (fracture Femur, Tibia or Tibia and Fibula both) from July 2012 to June 2014 under Spinal Anesthesia and Lumber Epidural Catheterization was collected. Thirty six patients were males and 21 were females. Patients were in between 17 to 91 years of age having ASA 1 and 2. All patients were premedicated with $1 \mathrm{mg}$ Lorazepam at night before surgery and kept nil per oral after midnight before the day of surgery. Informed written consent for surgery under anesthesia was taken from patients or from their relatives. Patients with ASA physical status more than 2 and those receiving intraoperative epidural anesthesia or analgesia and any other anesthetic or analgesic agents were excluded in this study.

Intravenous access was made with $18 \mathrm{G}$ cannula and Ringer lactate solution was infused in all patients. Monitor like Electrocardiography, Non-Invasive Blood Pressure and Pulse Oximetry was attached for continuous monitoring of vitals. After recording the baseline vitals, positioning of the patient for anesthesia was according to their choice either in lateral decubitus or in sitting position. Part was prepared with all aseptic technique. Two precent lignocaine $3 \mathrm{ml}$ was used to infiltrate the skin. In lateral decubitus with the operated limb below, Spinal Anesthesia was given using 0.5\% Heavy Bupivacaine 2.5 to $3 \mathrm{ml}$ (12.5mg to $15 \mathrm{mg}) 25 \mathrm{G}$ Quinke spinal needles through lower lumber intervertebral space. Then $18 \mathrm{G}$ Touhy Epidural needle was inserted one space above or below the space in which Spinal Anesthesia was given. Epidural space was reached with the loss of resistance to air technique by using $10 \mathrm{ml}$ syringe available inside the Epidural kit set. Distance between the Epidural space and the skin was noted and catheter was inserted through the needle to the epidural space. Length of the catheter at skin was also noted. $3 \mathrm{ml}$ of $2 \%$ Lignocaine with Adrenaline (1:200000) was given as a "Test Dose" via epidural catheter to rule out injection into vessel or sub-arachnoid space. Two small round coils of catheter were made around the insertion site. Small sterile gauze was placed to cover the coils and adhesive tape was applied to fix the catheter. In sitting position, epidural catheter was inserted before giving Spinal Anesthesia. Urinary catheterization was done in all patients of femur fracture. Throughout intraoperative period, vitals were monitored.

After completion of surgery, patients were kept in Post Anesthesia Care Unit (PACU) for an hour under continuous monitoring of vitals and then shifted to the postoperative ward on the next day. The written instruction was given mentioning not to give any analgesia to the patients and pain will be taken cared by the residents of Anesthesiology Department. Residents of Anesthesiology followed Departmental Protocol for epidural analgesia.

According to the Protocol, epidural top up contains $1 \mathrm{mg}$ preservative free Morphine and $2 \mathrm{ml}$ of $0.5 \%$ plain Bupivacaine diluted with Normal Saline to make total volume of $10 \mathrm{ml}$. First top up is given just after admission in PACU and then 12 hourly. If patient complains of pain before epidural top up time in postoperative period, injection ketorolac $30 \mathrm{mg}$ is given intravenously and epidural top up is also done immediately. Time of drug given is recorded. And on $3^{\text {rd }}$ postoperative day, Epidural catheter is removed 2 hours after the first epidural top up for this day and observations for pain or any complications is recorded for 24 hours after the last epidural top up.

\section{RESULTS}

It was found that injection Mephenteramine was used to treat hypotension for around 20 minutes after Spinal Anesthesia as intermittent boluses of $3 \mathrm{mg}$ in most of the patients. 
Bradycardia was not recorded in any cases. Injection Midazolam 1 to $2 \mathrm{mg}$ was given for all patients intraoperatively. Volume of Ringer Lactate given throughout the surgery was 2-2.5 liters. Colloid was not used in any patients. There were no records of intraoperative and postoperative blood and blood products transfusion. Dural tap had not occurred in any cases. Epidural test dose was not repeated before Epidural Top up in any patients. All patients were awake and responded to commands before shifting from OT. At PACU, no any significant hemodynamic alterations were recorded. Neither any medications were used nor oxygen supplemented. In postoperative ward, complications like hypotension, respiratory depression, nausea, vomiting, constipation, pruritus, motor blockade and infection at the catheter insertion site was also not present in any patients throughout the study period. All patients were fully awake and no any critical events were recorded throughout the study period. Not a single case of accidental Epidural catheter removal or blockade was found. Dressings were also not done at the Epidural catheter insertion sites. Urinary retention could not be noted because of catheterization in all patients with fracture Femur which was removed on $1^{\text {st }}$ or $2^{\text {nd }}$ postoperative day. There were no any records of giving prophylaxis for Deep Vein Thrombosis in any of the patients.

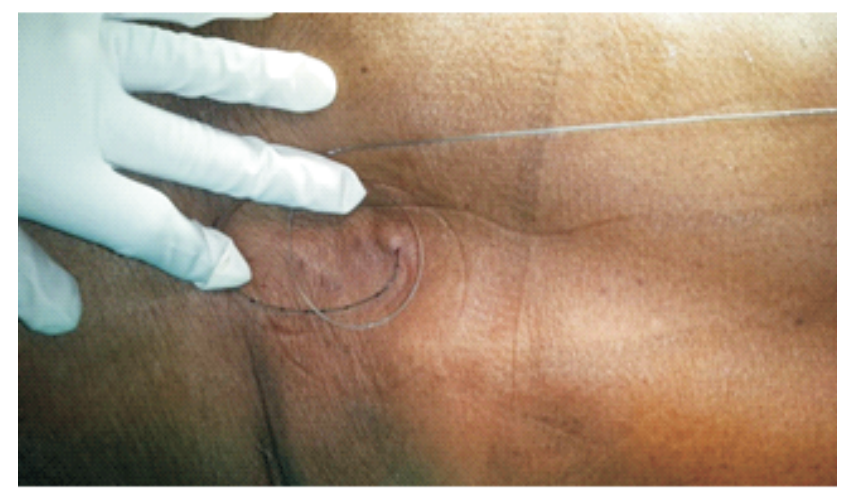

\section{Figure 1: Coils of Lumber Epidural Catheter}

All patients received epidural top up doses at 12 hour gap except in 2 patients. Out of them, 1 patient received 1 hour and another patient received 35 minutes before the expected top up time on the operation day. After this day, none of these patients received rescue analgesic for average 21.93 hours of last epidural top up. All patients were able to mobilize around their bed on $2^{\text {nd }}$ postoperative day. The average postoperative stay in hospital was 6.5 days.

\section{DISCUSSION}

The challenge of modern anesthesia and perioperative medicine is to create efficient treatment regimens with an optimal balance between protective and unwanted effects, in order to ensure patient safety and comfort, and to facilitate recovery. Surgical pain results in stress responses (endocrine, metabolic and inflammatory) not only intraoperatively but also in postoperative period. Reduction in surgical stress responses will lead to a reduced incidence of postoperative organ dysfunction and thereby to an improved outcome. There are several techniques for postoperative pain management and choice depends on several factors such as Anesthetist's experience, preference, duration of local and systemic pain management, contraindications of some analgesic drugs and techniques and patient's preferences. ${ }^{2}$

Though epidural analgesia technique is widely used to relieve postoperative pain, it carries a potential risk and limitations such as dural perforation, epidural hematoma and infection etc. ${ }^{3}$ The most commonly used opioid is Morphine and the most commonly used local anesthetic is Bupivacaine. Neuraxial opioids have both presynaptic and postsynaptic effects in the dorsal horn and affect the modulation of nociceptive input but do not cause motor or sympathetic blockade. Neuraxial local anesthetic results in sympathetic, sensory and motor blockade too.

Several studies ${ }^{4,5,6}$ with different doses of epidural morphine ranging from 0.5 to $10 \mathrm{mg}$ doses were studied to find its effectiveness and side effects in patients underwent cesarean section, femoral-popliteal bypass or total knee replacement and after other lower extremity surgeries. These studies found no any difference in pain relief and patient satisfaction with lower doses as compared to higher doses. They found statistically significant nausea and vomiting with $8 \mathrm{mg}$ doses whereas late respiratory depression was observed with $10 \mathrm{mg}$ doses only. Minor complications like nausea, vomiting, pruritus and urinary retention were uncommon and did not appear to be related to dose. These studies concluded that low doses are effective with minimal side effects. Chaney MA ${ }^{7}$ also reported four classic side effects of intrathecal and epidural opioids being pruritus, nausea and vomiting, urinary 
retention and respiratory depression. But, he found most of the side effects are dose dependent and are more common with intrathecal opioid.

A study done by Stenseth and colleagues ${ }^{8}$ using epidural morphine in 1085 postoperative patients found 90\% of patients underwent hip arthroplasty, prostatectomy, thoracotomy, major lower extremity surgery and laparotomy were completely satisfied with the postoperative course. Side effects like pruritus in $11 \%$, nausea or vomiting in $34 \%$ and respiratory depression (occurred as a gradually decreasing respiratory rate) in $0.9 \%$ of the total patient population was found whereas urinary retention occurred in $42 \%$ of patients not having urinary catheters in place. Here, the total dose of epidural morphine from the end of the surgery until the next morning varied from 4 to $18 \mathrm{mg}$. Similarly, Shafiq F and colleagues $^{9}$ collected the records of 1706 patients with epidural catheter for postoperative pain relief and found that the overall incidence of the complication was $26.6 \%$. The common complications were motor block (13.4\%), dural tap $(1.2 \%)$, ineffective pain control $(2.4 \%)$, accidental catheter pull outs $(3.8 \%)$ and problems associated with the delivery system of drug (1.7\%). The $12 \%$ of patients required intervention for the particular complications. The regime was discontinued in $28 \%$, drug concentration changed in $21.5 \%$ while the other modes of pain management were used in $19 \%$ of patients. $0.9 \%$ of patients required epidural blood patch while $2 \%$ of patients required catheterization for urinary retention. But, this retrospective study did not show any such complications.

Reiz S and colleagues ${ }^{10}$ found that total dose required in epidural group was $3.6 \mathrm{mg}$ and in intramuscular group was $41 \mathrm{mg}$ during 15 hour observation after hip surgery. It has been found that bolus epidural opioids produce longer lasting analgesia with smaller doses than intermittent Intramuscular opioids but there is less convincing evidence that they improve the quality of analgesia when the technique is compared to intravascular patient-controlled anaesthesia (PCA). In a meta- analysis on epidural versus systemic analgesia in various surgical procedures, epidural local anesthetic were found to reduce postoperative complications compared with epidural or systemic opioid techniques. ${ }^{11}$

Continuous infusion of local anesthetic or opioid alone or in combination provide effective postoperative pain relief but the most effective ones alone or in combination, dose and route remain controversial. ${ }^{12}$ Epidural local anesthesia or local anesthesia opioid mixtures or epidural opioid based regimens have demonstrated reduction in postoperative pulmonary or cardiac morbidity, reduction in postoperative paralytic ileus as well as improved postoperative cognitive function in elderly patients in various surgical procedures. ${ }^{13-24}$ Study has also found that continuous epidural local anesthetic reduces the risk of thromboembolic complications after lower body procedures but not after major abdominal procedures. ${ }^{13}$

Both local anesthetics and opioids individually cause their own characteristic side effects. The two drugs act by different mechanisms and so a combination of both types of drugs should lead to synergism of effect, a reduced dose of each drug and therefore fewer side effects. But, evidence from studies is controversial. The addition of 0.1 to $0.125 \%$ bupivacaine to epidural morphine may or may not prolong the duration of postoperative analgesia compared with morphine alone. ${ }^{25-28}$ Some involved bolus injections only and here the long duration of action of morphine compared with local anesthetics make the comparisons difficult. Fentanyl also gave similar confusing results, either no synergism or some benefit. ${ }^{29-31}$ Local anesthetic in higher concentration or higher infusion rates also showed some benefits. ${ }^{32,33}$ In above studies, the assessment of postoperative pain is often by different techniques and these need to be standardized before valid comparisons are made. In another meta-analysis of postoperative pain, $\mathrm{Wu}$ et al. ${ }^{34}$ compared effectiveness of systemic opioid, epidural opioid and epidural opioid-local anesthetic mixture. For all pain types, they found that epidural opioid was more effective than the systemic opioid and continuous epidural infusion was found more effective in pain at rest and movement than PCA. They also mentioned that epidural opioid mixed with local anesthetic was the best analgesic.

The combination of LA-opioid is commonly used and is based on the clinical observation that this combination limits the regression of the sensory block seen with LAs alone and improves the quality of dynamic pain relief. In majority of studies, use of LA-opioid mixture is associated with significantly better dynamic pain relief after lower and upper abdominal, orthopedic and thoracic surgery than the components of the mixture infused alone. ${ }^{35}$ This study also 
used a mixture of Bupivacaine and preservative free morphine which was found very effective in relieving postoperative pain. Hydrophilic opioid like morphine if given intrathecally has increased tendency to remain in CSF resulting in possible cephalad spread and delayed respiratory depression. Here, morphine was given epidurally in low dose. The records in this study did not find any patient suffering from respiratory depression or need of supplemental oxygen postoperatively. Most of the studies on postoperative epidural analgesia have used patient controlled analgesia technique or as a continuous infusion technique. In this retrospective study, intermittent bolus was given mostly 12 hour apart via Lumber Epidural Catheter by the anaesthesia residents. Literature with this technique is lacking and more studies are required to find the effectiveness of these drugs in this concentration and dose. This technique may be very beneficial in the developing countries like ours where PCA or infusion devices are not easily available.

\section{CONCLUSION}

Postoperative epidural analgesia is very safe and effective in relieving postoperative pain after major orthopedic surgery when mixture of morphine and bupivacaine is used as intermittent boluses 12 hourly. This technique might be very useful in the developing countries like ours where PCA devices are not easily available.

\section{ACKNOWLEDGEMENT}

I would like to thank Prof. Dr. P.B.Oram, Head, Department of Anesthesiology, UCMS Anesthesiology, UCMS for his continuous support and my residents for helping me in collecting the data.

\section{REFERENCES}

1) Kehlet H. Multimodal approach to control postoperative pathophysiology and rehabilitation. Br. J Anaesth 1997; 78: 606-17

2) Savage C, McQuitty C, Wang D et al - Postthoracotomy pain management. Chest Surg Clin NAm, 2002; 12(2):251-63.

3) Andreas M, Zollinger A, De Lorenzi D et al. - Prospective, randomized comparison of extrapleural versus epidural analgesia for postthoracotomy pain. Ann Thorac Surg, 1998; 66:367-72.
4) Chumpathong S1, Santawat U, Saunya $P$, Chimpalee $R$, Toomtong P. Comparison of different doses of epidural morphine for pain relief following cesarean section. J Med Assoc Thai. 2002 Sep;85 Suppl 3:S956-62.

5) Allen PD, Walman T, Concepcion M, Sheskey M, Patterson $M K$, Cullen D, Covino BG. Epidural morphine provides postoperative pain relief in peripheral vascular and orthopedic surgical patients: a dose-response study. Anesth Analg. 1986 Feb;65(2):165-70.

6) Martin $R$, Salbaing $J$, Blaise $G$, Tétrault JP, Tétreault $L$. Epidural morphine for postoperative pain relief: a doseresponse curve. Anesthesiology. 1982 Jun;56(6):423-6.

7) Chaney MA. Side effects of intrathecal and epidural opioids. Can JAnaesth. 1995 Oct;42(10):891-903.

8) Stenseth $R$, Sellevold $O$, Breivik H. Epidural morphine for postoperative pain: experience with 1085 patients. Acta Anaesthesiol Scand. 1985 Jan;29(1):148-56.

9) Shafiq F1, Hamid M, Samad K. Complications and interventions associated with epidural analgesia for postoperative pain relief in a tertiary care hospital. Middle East J Anaesthesiol. 2010 Oct; 20(6):827-32.

10) Reiz S, Ahlin J, Ahrenfeldt B, Andersson M, Andersson $S$ Epidural morphine for postoperative pain relief. Acta Anaesthesiol Scand. 1981 Apr;25(2):111-4.

11) Ballantyne JC, Carr DB, de Ferranti S, Suarez T, Lau J, Chalmers TC, Angelillo IF, Mosteller F. The comparative effects of postoperative analgesic therapies on pulmonary outcome: cumulative meta-analysis of randomized, controlled trials. Anesth Analg 1998; 86: 598-612.

12) Glass PSA, EstokP, Ginsberg B, Goldberg JS, Sladen RN Use of patient controlled analgesia to compare the efficacy of epidural to intravenous fentanil administration. Anesth Analg, 1992; 74:345-351.

13) Kehlet $H$ and Holte K. Effect of postoperative analgesia on surgical outcome. Br J Anaesth 2001; 87: 62-72.

14) Veering BT, Cousins MJ - Cardiovascular and pulmonary effects of epidural anaesthesia. Anaesth Intens Care, 2000;28:620-635.

15) Carli F, Trudel J, Belliveau P. Return of bowel function after thoracic epidural anesthesia. Dis Colon Rectum 2001.

16) Hijorto NC, Neumann P, Frosig F, et al. A controlled study on the effect of Epidural analgesia with local anaesthetics and morphine on morbidity after abdominal surgery. Acta Anaesthesiol Scand 1985; 29: 790-6 
17) Jayr C, Thomas H, Rey A, Farhat F, Lasser P, Bourgain JL. Postoperative pulmonary complications. Epidural analgesia using bupivacaine and opioid versus parentral opioid. Anesthesiology 1993; 78: 666-76

18) Liu S, Carpenter RL, Mackey DC, et all. Effects of postoperative analgesic technique on rate of recovery after colon surgery. Anesthesiology 1995; 83: 757-65

19) Mann C, Pouzerattee Y, Boccara G, et al. Comparision of intravenous or epidural patient-controlled analgesia in the elderly after major abdominal surgery. Anesthesiology 2000, 92: 433-41

20) Seeling W, Bothner U, Eifert B, et all. Patient-controlled analgesia versus epidural analgesia using bupivacaine or morphine following major abdominal surgery. No difference in postoperative morbidity. Anaesthesist 1991; 40: 614-23

21) Seeling W, Bruckmooser KP, Hufner C, Kneitinger E, Rigg C, Rockemann $M$. No reduction in postoperative complications by the use of catheterized epidural analgesia following major abdominal surgery. Anaesthesist 1990; 39: 33-40

22) Holte K, Kehlet H. Epidural anesthesia and risk of anastomotic leakage. Reg Anesth Pain Med 2001; 26: 111-7

23) Christopherson R, Beattie C, Frank SM, et al. Perioperative Ischemia Randomized Anesthesia Trial study Group. Perioperative morbidity in patients randomized to epidural or general anesthesia for lower extremity vascular surgery. Anesthesiology 1993; 79: 422-34

24) Yeager MP, Glass DD, Neff RK, Brinck-Johnsen T. Epidural anesthesia and analgesia in high risk surgical patients. Anesthesiology 1987; 66: 729-36

25) Hanson, A.L., Hanson, B., Matousek, M., 1984. Epidural anesthesia for cesarean section. The effect of morphinebupivacaine administered epidurally for intra and postoperative pain relief.. Acta Obstet Gynecol Scand, Acta Obstet Gynecol Scand 63, 135-40.

26) Cullen, M.L., Staren, E.D., Ganzouri, Ael, Logas, W.G., Ivankovitch, A.D., Economov, S.G., 1985. Continuous epidural analgesia after major abdominal surgery.. Surgery, Surgery 98, 718-28.

27) Logas, W.G., el-Baz, N., el-Ganzouri, A., Cullen, M., Staren, E., Faber, L.P., Ivankovich, A.D., 1987. Continuous thoracic epidural analgesia for postoperative pain relief following thoracotomy: a randomized prospective study. Anesthesiology. Anesthesiology 67, 787-91.

28) Douglas, M.J., McMorland, G.H., Janzen, J.A., 1988. Influence of bupivacaine as an adjuvant to epidural morphine for analgesia after cesarean section.. Anesth Analg, Anesth Analg $67,1138-41$
29) Badner, N.H., Sandler, A.N., Koren, G., Lawson, S.L., Klein, J. Einarson, T.R., 1990. Lumbar epidural fentanyl infusions for post-thoracotomy patients: analgesic, respiratory, and pharmacokinetic effects. J Cardiothorac Anesth, J Cardiothorac Anesth 4, 543-51.

30) Fischer, R.L., Lubenow, T.R., Liceaga, A., McCarthy, R.J., Ivankovich, A.D., 1988. Comparison of continuous epidural infusion of fentanyl-bupivacaine and morphine-bupivacaine in management of postoperative pain.. Anesth Analg, Anesth Analg 67, 559-63.

31) Lubenow, T.R., Wong, J., McCarthy, R.J., Logas, W.G., Ivankovich, A.D., 1988. Prospective evaluation of continuous epidural narcotic-bupivacaine infusions in 1000 patients. Anesthsiology, Anesthsiology 69, 389+.

32) Lee, A., Simpson, D., Whitfield, A., Scott, D.B., 1988. Postoperative analgesia by continuous extradural infusion of bupivacaine and diamorphine.. Br J Anaesth, Br J Anaesth 60, 845-50.

33) Elliot, R.D., 1991. Continuous infusion of epidural analgesia for obstetrics: Bupivacaine versus Bupivacaine-Fentanyl mixture.. Canadian Journal of Anesthesia, Canadian Journal of Anesthesia 38, 303-10.

34) Wu CL, Cohen SR, Richman JM et al. - Effi cacy of postoperative patient-controlled and continuous infusion epidural analgesia versus intravenous patient-controlled analgesia with opioids: a meta-analysis. Anesth, 2005;103(5):1079-88.

35) RG Wheatley, S. A. Schug and D Watson. Safety and efficacy of postoperative epidural analgesia. BrJ Anaesth 2001; 87: 47-61 